TUMOUR IMMUNITY

\title{
Joint effort needed
}

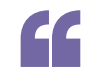

\section{tumours \\ modified to \\ express both \\ the MHC class I \\ and class II \\ neoantigens \\ were rejected}

Much of the research on cancer immunotherapy has focused on eliciting $\mathrm{CD}^{+} \mathrm{T}$ cells that kill cancer cells. But evidence is mounting that $\mathrm{CD} 4^{+} \mathrm{T}$ cells are also required and may be crucial determinants of a successful response to immunotherapy, as reported by Robert Schreiber and colleagues in Nature.

Our understanding of the involvement of $\mathrm{CD}^{+} \mathrm{T}$ cells in antitumour immunity has been limited by a paucity of known tumour-derived neoantigens recognized by $\mathrm{CD} 4^{+}$ $\mathrm{T}$ cells. Schreiber and colleagues use a new predictive algorithm to identify MHC class II-binding peptides from the T3 sarcoma cell line. They calculated the likelihood of each of the 700 missense mutations in T3 sarcoma cells being presented by MHC class II, on the basis of binding affinity and transcript levels. They identified one point mutation in $\beta 1$ integrin (mITGB1) that fit all of the criteria for a MHC class II-binding neoantigen and activated $\mathrm{CD} 4^{+} \mathrm{T}$ cells in vitro.

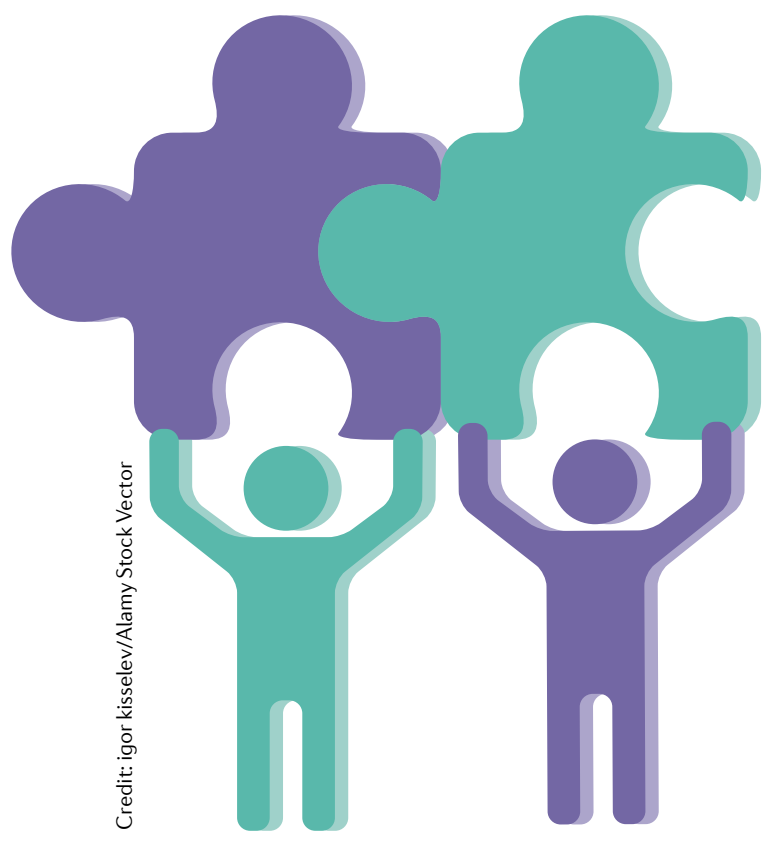

MHC class II tetramers generated with the mITGB1 peptide stained a significant subset of $\mathrm{CD} 4^{+}$tumourinfiltrating lymphocytes from mice bearing T3 tumours compared with control tetramers. The mITGB1specific $\mathrm{CD} 4^{+} \mathrm{T}$ cells were only activated by mITGB1 and not wildtype ITGB1, and they had a T helper 1 cell phenotype.

So, do these tumour-specific $\mathrm{CD} 4^{+}$ $\mathrm{T}$ cells have a role in tumour rejection? A nonimmunogenic sarcoma cell line (KP9025) was engineered to express a known T3 sarcoma-derived MHC class I neoantigen (mutated laminin- $\alpha 4$; mLAMA4) and the MHC class II neoantigen mITGB1 and injected into mice. Whereas unmodified KP9025 tumours grew progressively, tumours modified to express both the MHC class I and class II neoantigens were rejected. Expression of either neoantigen alone was not sufficient to induce immunogenicity even in the presence of immune checkpoint blockade. This suggests that a combined response by both $\mathrm{CD} 8^{+}$and $\mathrm{CD} 4^{+} \mathrm{T}$ cells is required for tumour rejection.

To determine how $\mathrm{CD} 4^{+} \mathrm{T}$ cells 'help' CD8 ${ }^{+} \mathrm{T}$ cells, the authors measured cytotoxicity of $\mathrm{CD}^{+} \mathrm{T}$ cells in tumour-bearing mice. Mice bearing KP9025 sarcomas expressing only the MHC class I neoantigen mLAMA4 were largely incapable of eliminating mLAMA4-peptide-pulsed splenocytes in vivo. However, mice with sarcomas expressing both MHC class I and II neoantigens efficiently eliminated mLAMA4-pulsed splenocytes but not splenocytes pulsed with an irrelevant peptide. These mice also had increased numbers of mLAMA4specific $\mathrm{CD}^{+} \mathrm{T}$ cells. Together, the results suggest that $\mathrm{CD} 4^{+} \mathrm{T}$ cells help support $\mathrm{CD}^{+} \mathrm{T}$ cell priming and cytotoxic activity.
So could a vaccine consisting of the MHC class I and II neoantigens protect against challenge with T3 sarcoma cells? Vaccination of naive mice with irradiated unmodified KP9025 cells or KP9025 cells expressing either mLAMA4 or mITGB1 was not sufficient to protect from subsequent tumour challenge. By contrast, mice that received a vaccine containing cells expressing both mLAMA4 and mITGB1 had higher levels of interferon- $\gamma$ (IFN $\gamma$ )-producing $\mathrm{CD}^{+} \mathrm{T}$ cells and more efficiently prevented tumour outgrowth.

Further experiments showed that expression of MHC class II neoantigens was required in the tumour site. They implanted mice with tumour cells expressing only mLAMA4 on one flank and tumour cells expressing both mLAMA4 and mITGB1 on the opposite flank, and treated the mice with immunotherapy. As expected, the tumour that expressed both antigens regressed, but the tumour that expressed only mLAMA4 continued to grow slowly.

Finally, the authors noted a marked influx of macrophages expressing inducible nitric oxide synthase (iNOS) in the regressing tumours compared with the growing tumours. This effect required the presence of both $\mathrm{CD}^{+}{ }^{+} \mathrm{T}$ cells and $\mathrm{CD} 8^{+} \mathrm{T}$ cells in the tumour, where they may support iNOS expression by macrophages through the production of IFN $\gamma$.

This study promises to stimulate further investigation into the antitumour $\mathrm{CD}^{+} \mathrm{T}$ cell response, in particular to determine whether the presence of $\mathrm{CD} 4^{+} \mathrm{T}$ cells can predict responsiveness to immunotherapy in patients with cancer.

Lucy Bird

ORIGINAL ARTICLE Alspach, E. et al. MHC-II neoantigens shape tumour immunity and response to immunotherapy. Nature https:// doi.org/10.1038/s41586-019-1671-8 (2019) RELATED ARTICLE Linehan, J. L. \& Delamarre, L. Teamwork by different T-cell types boosts tumour destruction by immunotherapy. Nature https:// doi.org/10.1038/d41586-019-03106-1 (2019) 